\title{
Spikes in cosmic crystallography II: topological signature of compact flat universes
}

\author{
G.I. Gomero*, M.J. Rebouças ${ }^{\dagger}$, \& A.F.F. Teixeira ${ }^{\ddagger}$ \\ Centro Brasileiro de Pesquisas Físicas, \\ Rua Dr. Xavier Sigaud 150, \\ 22290-180 Rio de Janeiro - RJ, Brasil
}

June 26, 2021

\begin{abstract}
We study the topological signature of euclidean isometries in pair separations histograms (PSH) and elucidate some unsettled issues regarding distance correlations between cosmic sources in cosmic crystallography. Reducing the noise of individual PSH's using mean pair separations histograms we show how to distinguish between topological and statistical spikes. We report results of simulations that evince that topological spikes are not enough to distinguish between manifolds with the same set of Clifford translations in their covering groups, and that they are not the only signature of topology in PSH's corresponding to euclidean small universes. We also show how to evince the topological signature due to non-translational isometries.
\end{abstract}

\section{Introduction}

The method of Cosmic Crystallography (CC), devised by Lehoucq et al. [1], looks for distance correlations between cosmic sources using pair separations histograms (PSH), i.e.

\footnotetext{
*E-mail: german@cbpf.br

${ }^{\dagger}$ E-mail: reboucas@cbpf.br

${ }^{\ddagger}$ E-mail: teixeira@cbpf.br
} 
plots of the number of pairs of sources versus the distance (or squared distance) between them. These correlations arise from the isometries of the covering group of the 3-manifold used to model our Universe and so they provide a signature of its global spatial topology. In this way $\mathrm{CC}$ is potentially useful to investigate the shape and size of our Universe. It has recently been shown by Gomero et al. [2] how to calculate the topological signature from these distance correlations in a very general geometrical-topological-observational setting. It turns out from the major result of Ref. [2] that correlations due to Clifford translations manifest as spikes in PSH's, whereas other isometries manifest as small deformations of the expected pair separations histogram (EPSH) of the corresponding universal covering manifold.

The major result of Ref. [2] has a striking consequence for universe models with hyperbolic spatial sections. Indeed, since no hyperbolic isometry is a Clifford translation, there are no topological spikes in PSH's corresponding to low density universe models. Thus, at first sight, these histograms seem to give no reliable information of the topology of the spatial sections in these models of the universe. The absence of spikes in PSH's from hyperbolic universes is by now well understood and has been confirmed by simulations performed by Lehoucq et al. [4] and Fagundes and Gausmann [5]. It remains, however, to understand the topological signature of hyperbolic isometries in CC.

The implications of the results of Ref. [2] for PSH's from flat universe models seem to be less well understood. It has been stated in [4] and [6] that every euclidean isometry which produces $\Gamma$-pairs in a given catalog will give rise to a spike in the corresponding PSH. This statement, however, is in clear contradiction with the fact that only translations produce spikes [2]. Moreover, in studying the applicability of $\mathrm{CC}$ to closed flat models of our Universe, Fagundes and Gausmann [7] reported a PSH for a manifold of class $\mathcal{G}_{6}$, therein called model $E 4$, which exhibits a significant peak at $(d / L)^{2}=5$. That paper suggests that this spike is generated by an isometry of the covering group of the manifold considered, and this interpretation was again suggested in Ref. [5]. Nevertheless, according to Ref.[2], since there is no translation that would produce the peak at $(d / L)^{2}=$ 5 , one immediately concludes that it must be due to statistical fluctuations, and so it is not of topological origin. A definitive elucidation of these unsettled issues would be useful because it would clarify the actual signature of euclidean non-translational isometries in PSH's. Indeed, by performing simulations we will evince in this letter that, contrary to what is suggested in [4] and [6], topological spikes are not the only signature of topology in PSH's corresponding to euclidean small universes. Besides we also show

\footnotetext{
${ }^{1}$ In this letter we use the notation of Ref. [8] to denote families of flat compact orientable 3-manifolds.
} 
through simulations that non-translational isometries do not manifest as less sharp peaks as suggested by Fagundes and Gausmann [5], but as broad and tiny deformations of the PSH corresponding to the simply connected case. Our results here are supported by and in agreement with the general theoretical developments of Ref. [2].

Actually, the major purpose of this letter is to show how to use the MPSH technique described in [2] for studying the topological signature of isometries in PSH's. After a brief review of the techniques developed in [2] we first compute MPSH's for a manifold $M$ of class $\mathcal{G}_{6}$ and reduce the statistical noise to a level that allows the identification of topological spikes. In this way (i) statistical spikes that may be confused with topological spikes are removed, and (ii) topological spikes that are masked by statistical fluctuations in individual PSH's show up even when there are few $\Gamma$-pairs corresponding to them. Incidentally, point (i) makes clear that actually there is no topological spike at $(d / L)^{2}=5$. As an additional application we construct an EPSH for the minimal 3-torus that covers $M$ and plot the difference between this EPSH and an MPSH of $M$. Since the covering groups of this 3-torus and that of $M$ have the same translations, then PSH's for these two manifolds would exhibit identical spike spectra [2]. So this difference yields the topological signature of non-translational isometries of the covering group of $M$ plus some statistical noise. For comparison, we also plot the difference between an MPSH and an EPSH, both for the 3-torus, obtaining as a result essentially statistical noise. This indicates that, within the accuracy of the simulations, topological spikes are the only topological signature in PSH's for a 3-torus.

\section{Pair separations histograms}

Here we briefly review some results obtained in Ref. [2], and extend them to the level needed for the development of this work. We begin by describing what a pair separations histogram (PSH) is, and then show how to construct mean pair separations histograms (MPSH) with simulated catalogs. We end with a brief explanation of the expected pair separations histogram (EPSH) and its use in determining the topological signature for non-translational isometries.

To build a PSH we simply evaluate a suitable one-to-one function $f$ of the separation $r$ of every pair of cosmic sources from a given catalog $\mathcal{C}$, and then count the number of pairs for which these values $f(r)$ lie within certain subintervals. These subintervals are all of equal length and must form a partition of the interval $(0, f(D)]$, where $D$ is the diameter of the observed universe corresponding to the catalog. A PSH is just a normalized plot 
of this counting. We will take the function $f$ to be the square function as is usual when dealing with flat models in $\mathrm{CC}$, in order to compare our plots with those found in the literature.

It is convenient to have a formal description of the above procedure. In considering discrete astrophysical sources, the observable universe can be viewed as that part of the universal covering manifold $\widetilde{M}$ of the space-like section $M$ of spacetime, causally connected to an image of our position since the moment of matter-radiation decoupling; while, given a catalog of cosmic sources, the observed universe $\mathcal{U}$ is that part of the observable universe which contains all the sources listed in the catalog. So, for instance, the observed universe corresponding to a catalog covering the entire sky is a ball of radius given by the redshift cutoff of the catalog, while for a pencil beam catalog it is a thin cone with vertex at an image of our position. Interesting observed universes that may be explored in the context of CC are thin spherical shells; these observed universes correspond to catalogs with approximately equal upper and lower redshift cutoffs [2].

All the sources contained in $\mathcal{U}$ can be observed in principle, but due to observational limitations a catalog consists only of part of them. Our observational limitations can be formulated as selection rules which describe how the catalog arises from the set of observable images. These selection rules, together with the distribution law which the objects in $M$ obey, will be referred to as construction rules for the catalog $\mathcal{C}$. It should be noted that the above definition for a catalog fits in with the two basic types of catalogs one usually finds in practice, namely real catalogs (which arise from observations) and simulated catalogs, which are generated under well-defined assumptions that are posed to mimic some observational limitations and (or) to account for simplifying hypotheses.

To construct a PSH one begins by dividing the interval $\left(0, D^{2}\right]$ in $m$ equal subintervals of length $\Delta s=D^{2} / m$. Each subinterval has the form

$$
J_{i}=\left(s_{i}-\frac{\Delta s}{2}, s_{i}+\frac{\Delta s}{2}\right] \quad ; \quad i=1,2, \ldots, m
$$

and is centered at

$$
s_{i}=\left(i-\frac{1}{2}\right) \Delta s
$$

Given a catalog $\mathcal{C}$ of cosmic sources and denoting by $\eta(s)$ the number of pairs of sources in $\mathcal{C}$ with squared separation $s$, a PSH is then obtained plotting the function

$$
\Phi\left(s_{i}\right)=\frac{2}{N(N-1)} \frac{1}{\Delta s} \sum_{s \in J_{i}} \eta(s)
$$

where $N$ is the number of sources in $\mathcal{C}$. Note that with the same catalog $\mathcal{C}$ we may obtain different PSH's simply by taking different values for $m$. The sum in (2.1) is just 
a counting and the coefficient of the sum is a normalization constant, so

$$
\sum_{i=1}^{m} \Phi\left(s_{i}\right) \Delta s=1
$$

Although it is usual in CC to refer to the plot of the function $\Phi\left(s_{i}\right)$ as a PSH, for theoretical purposes it is more useful to define a PSH as a function given by (2.1). From now on we will always refer to $\Phi\left(s_{i}\right)$ simply as a PSH.

Any single PSH is plagued with statistical noise that may mask the topological signature. The simplest and most obvious way to reduce this noise is to use the MPSH which is described as follows. Consider $K$ comparable catalogs $\mathcal{C}_{k}(k=1,2, \ldots, K)$, with approximately the same number of cosmic sources and corresponding to the same manifold $M$. Let their PSH's, for a fixed value of $m$, be given by

$$
\Phi_{k}\left(s_{i}\right)=\frac{2}{N_{k}\left(N_{k}-1\right)} \frac{1}{\Delta s} \sum_{s \in J_{i}} \eta_{k}(s),
$$

where $N_{k}$ is the number of sources in $\mathcal{C}_{k}$ and $\eta_{k}(s)$ is the number of pairs of sources in $\mathcal{C}_{k}$ with squared separation $s$; then, the MPSH defined by

$$
<\Phi\left(s_{i}\right)>=\frac{1}{K} \sum_{k=1}^{K} \Phi_{k}\left(s_{i}\right)
$$

contains much less noise than any single PSH, and clearly contains the same topological information. Indeed, elementary statistics tells us that the statistical fluctuations in the MPSH are reduced by a factor proportional to $1 / \sqrt{K}$, which makes at first sight the MPSH very attractive. In Sec. 3 we apply this technique to discriminate between topological and statistical spikes in PSH's corresponding to an euclidean compact manifold.

As shown in Ref. [2] (see also Refs. [3, 9]), in the limit $K \rightarrow \infty$ the MPSH approximates very well to the EPSH which is an "ideal" PSH, i.e. a PSH with the statistical noise completely removed. Equation (4.15) of Ref. [2] [or equivalently eq. (2.11) rederived in Ref. [3], wherein $N=n(n-1) / 2$ denotes the total number of pairs of cosmic images] can be rewritten in the form

$\Phi_{\text {exp }}\left(s_{i}\right)=\Phi_{\text {exp }}^{s c}\left(s_{i}\right)+\frac{\nu_{u}}{N-1}\left[\Phi_{\text {exp }}^{u}\left(s_{i}\right)-\Phi_{\text {exp }}^{s c}\left(s_{i}\right)\right]+\frac{1}{N-1} \sum_{g \in \widetilde{\Gamma}} \nu_{g}\left[\Phi_{\text {exp }}^{g}\left(s_{i}\right)-\Phi_{\text {exp }}^{s c}\left(s_{i}\right)\right]$,

where $\widetilde{\Gamma}$ is the covering group $\Gamma$ of $M$ without the identity element, $N$ is the mean value of the $N_{k}, \Phi_{\text {exp }}^{s c}\left(s_{i}\right)$ is the EPSH of the corresponding simply connected case, $N_{u}$ is the expected number of uncorrelated pairs and $\nu_{u}=2 N_{u} / N$. In 2.5) $\Phi_{\text {exp }}^{u}\left(s_{i}\right)=F_{u}\left(s_{i}\right) / \Delta s$, where $F_{u}\left(s_{i}\right)$ is the probability of an uncorrelated pair to be separated by a squared 
distance that lies in $J_{i}$. For each covering isometry it is also defined a number $\nu_{g}=N_{g} / N$, where $N_{g}$ is the expected number of $g$-pairs in a catalog with $N$ sources, and a distribution function $\Phi_{e x p}^{g}\left(s_{i}\right)=F_{g}\left(s_{i}\right) / \Delta s$, where $F_{g}\left(s_{i}\right)$ is the probability of an observed $g$-pair to be separated by a squared distance that lies in $J_{i}$.

Now within the approximation

$$
\Phi_{\text {exp }}^{s c}\left(s_{i}\right) \approx \Phi_{\text {exp }}^{u}\left(s_{i}\right)
$$

the EPSH reads

$$
\Phi_{e x p}\left(s_{i}\right) \approx \Phi_{e x p}^{s c}\left(s_{i}\right)+\frac{1}{N-1} \sum_{g \in \widetilde{\Gamma}} \nu_{g}\left[\Phi_{e x p}^{g}\left(s_{i}\right)-\Phi_{e x p}^{s c}\left(s_{i}\right)\right] .
$$

The general underlying setting for performing the calculations involved in (2.7) is the assumed existence of an ensemble of catalogs comparable to a given catalog $\mathcal{C}$ (real or simulated), with the same number of sources and corresponding to the same manifold $M$. The construction rules permit the computation of probabilities and expected values involved in (2.7).

Let $\Gamma_{t} \subset \Gamma$ be the subset of all Clifford translations of $\Gamma$ (i.e. all the isometries $g \in \Gamma$ such that for all $p \in \widetilde{M}$, the distance $|g(p)|=d(p, g p)$ is independent of $p)$. When $g \in \Gamma_{t}$ we have

$$
\Phi_{\text {exp }}^{g}\left(s_{i}\right)=\frac{\delta_{i, i_{g}}}{\Delta s}
$$

where $\delta_{i, i_{g}}$ is the Kronecker delta, and $i_{g}$ is the position of the spike due to the translation $g \in \Gamma_{t}$, i.e. $|g|^{2} \in J_{i_{g}}$. Then one can write (2.7) as

$$
\Phi_{\text {exp }}\left(s_{i}\right) \approx \Phi_{\text {exp }}^{s c}\left(s_{i}\right)+\varphi_{\text {exp }}^{t}\left(s_{i}\right)+\varphi_{\text {exp }}^{n t}\left(s_{i}\right)
$$

where

$$
\varphi_{\text {exp }}^{t}\left(s_{i}\right)=\frac{1}{N-1} \sum_{g \in \widetilde{\Gamma}_{t}} \nu_{g}\left[\frac{\delta_{i, i_{g}}}{\Delta s}-\Phi_{\text {exp }}^{s c}\left(s_{i}\right)\right]
$$

is the contribution of Clifford translations to the topological signature of the EPSH, and

$$
\varphi_{\text {exp }}^{n t}\left(s_{i}\right)=\frac{1}{N-1} \sum_{g \in \Gamma \backslash \Gamma_{t}} \nu_{g}\left[\Phi_{e x p}^{g}\left(s_{i}\right)-\Phi_{e x p}^{s c}\left(s_{i}\right)\right]
$$

is the topological signature associated to the non-translational isometries of $\Gamma$.

It is clear from (2.9) that manifolds with the same translations in their covering groups will exhibit the same spike spectra given by (2.10), the only difference between their

\footnotetext{
${ }^{2}$ This approximation is justified a posteriori by Fig.5a for the case of a 3 -torus.
} 
EPSH's being the topological signature associated to non-translational isometries. Moreover, in the euclidean case, from (2.9) one can always write

$$
\varphi_{\text {exp }}^{n t}\left(s_{i}\right) \approx \Phi_{\text {exp }}\left(s_{i}\right)-\Phi_{\text {exp }}^{\text {torus }}\left(s_{i}\right)
$$

with

$$
\Phi_{\text {exp }}^{\text {torus }}\left(s_{i}\right) \approx \Phi_{\text {exp }}^{s c}\left(s_{i}\right)+\varphi_{\text {exp }}^{t}\left(s_{i}\right)
$$

being the EPSH of the minimal 3-torus that covers $M$.

Now, using the fact that $\left\langle\Phi\left(s_{i}\right)>\approx \Phi_{\exp }\left(s_{i}\right)\right.$ one gets another approximate expression for the topological signature of non-translational isometries of small flat universes, namely

$$
\varphi_{\text {exp }}^{n t}\left(s_{i}\right) \approx<\Phi\left(s_{i}\right)>-\Phi_{\text {exp }}^{\text {torus }}\left(s_{i}\right) .
$$

This expression can easily be numerically evaluated since the MPSH can be obtained with computer simulations, and $\Phi_{\text {exp }}^{\text {torus }}\left(s_{i}\right)$ is given explicitly by

$$
\Phi_{\text {exp }}^{\text {torus }}\left(s_{i}\right) \approx\left(1-\frac{1}{N-1} \sum_{g \in \widetilde{\Gamma}_{t}} \nu_{g}\right) \Phi_{e x p}^{s c}\left(s_{i}\right)+\frac{1}{\Delta s(N-1)} \sum_{g \in \widetilde{\Gamma}_{t}} \nu_{g} \delta_{i, i_{g}} .
$$

In Sec. 1 we use (2.14) and (2.15) to evince the shape of the topological signature of non-translational isometries for an euclidean closed manifold.

From now on we will consider only trivial construction rules, i.e. we will assume that cosmic sources are uniformly distributed in space, and all cosmic sources present in universe models, up to a given redshift, are recorded in catalogs. Although unrealistic, this assumption makes easy to illustrate the general results developed in Ref.[2] and permits a comparison with current literature in $\mathrm{CC}$. Besides, in this case we can readily compute $\Phi_{e x p}^{s c}\left(s_{i}\right)$ and the coefficients $\nu_{g}$.

Indeed, from Ref. 10 (see also Ref. [11) one can easily calculate $\Phi_{\text {exp }}^{s c}\left(s_{i}\right)$ for the case of trivial construction rules and a ball of radius $R$ as an observed universe. For flat models one obtains

$$
\Phi_{\text {exp }}^{s c}(s)=\frac{3}{32} \frac{\sqrt{s}}{R}\left(2-\frac{\sqrt{s}}{R}\right)^{2}\left(4+\frac{\sqrt{s}}{R}\right) \Theta\left(2-\frac{\sqrt{s}}{R}\right),
$$

where $\Theta$ is the Heaviside function. Correspondingly, the coefficients $\nu_{g}$ can be calculated by simple geometrical arguments. Indeed, let $\mathcal{U}$ be the observed universe, i.e. a ball of radius $R$ centered at our position. The isometry $g$ transforms isometrically the ball $g^{-1}(\mathcal{U})$ into the ball $\mathcal{U}$, so only the sources in $g^{-1}(\mathcal{U}) \cap \mathcal{U}$ have a $g$-partner in $\mathcal{U}$, and form $g$-pairs. Thus we have

$$
\nu_{g}=\frac{\operatorname{Vol}\left(g^{-1}(\mathcal{U}) \cap \mathcal{U}\right)}{\operatorname{Vol}(\mathcal{U})}
$$


A simple calculation yields

$$
\nu_{g}=1-\frac{3}{4}\left(\frac{d_{g}}{R}\right)+\frac{1}{16}\left(\frac{d_{g}}{R}\right)^{3},
$$

where $d_{g}$ is the distance from the center of the observed universe to its image by the isometry $g$.

\section{$3 \quad$ Topological and statistical spikes}

Let us now show how to use the MPSH technique to discriminate between topological and statistical spikes by working out two examples of models of the universe reported in Ref.[7]. In order to make a comparison with the plots of the upper part of fig.1 in Ref. [7], we took a manifold $M$ of type $\mathcal{G}_{6}$ with covering group $\Gamma$ generated by

$$
\begin{aligned}
& \alpha(x, y, z)=(x+1,-y,-z), \\
& \beta(x, y, z)=(-x, z+1, y), \\
& \delta(x, y, z)=(-x, z, y+1),
\end{aligned}
$$

where $(x, y, z) \in R^{3}$. A fundamental polyhedron for $M$ and a detailed construction of this manifold is given in Ref. [12] (see also [13]).

We have performed simulations for two observed universes with radii $R_{1}=\sqrt{2}$ and $R_{2}=5 / 6$, respectively. As in Ref. [7], for each simulation in the first case $\left(R_{1}=\sqrt{2}\right)$ we put 20 objects uniformly distributed in the $\mathrm{FP}$, while in the case $R_{2}=5 / 6$ we put 101 objects inside it. In both cases we end up with catalogs of approximately 240 sources. A PSH for one catalog for each case is shown in fig.1, where we have subdivided the intervals $\left(0,4 R_{i}^{2}\right]$ in bins of width 0.01 as in Ref.[0]. A direct comparison with the plots in Ref. [7] can be done by considering unit lengths of $4200 h^{-1} M p c$ for the first case and $7200 h^{-1} M p c$ for the second. An important difference between our plots and those in Ref. [7] is that ours are normalized histograms as described in Sec.8. We can see that the PSH in fig.1a presents no apparent spike at $s=5$, suggesting that the corresponding spike in Ref. [7] is of statistical origin. In order to elucidate this and related issues we shall now discuss the MPSH's corresponding to these PSH's.

Figure 2 shows one MPSH built with 50 computer generated comparable catalogs with approximately 240 sources for each case of study. It can be seen that, by taking the mean over 50 catalogs, the statistical noise has been considerably reduced. Moreover it became apparent from such plots that there is no topological spike at $s=5$ for the first model of the universe, whereas for the second there is a small spike at $s=2$ that is 
masked by statistical fluctuations in the individual PSH's of fig.1b of this work and fig.1 of Ref.[7]. Both MPSH's show the agreement between our simulations and the theoretical results of [2]. Besides, it can be shown that there are no topological spikes at any odd integer position in PSH's corresponding to models of the universe which have $M$ as spatial sections of spacetime (see Appendix A for details).

\section{Topological signature of non-translational isome- tries}

Let us now consider the group $\Gamma_{t}$ generated by the translations $a=\alpha^{2}, b=\beta^{2}$ and $c=\delta \beta^{-1}$. This group is formed by all the translations in $\Gamma$ and is the covering group of the minimal 3-torus that covers $M$. A fundamental polyhedron for this 3-torus is a parallelepiped of height 2 and square base of side $\sqrt{2}$. As it has been shown in Sec.2, PSH's corresponding to models of the universe that have the same translations in their covering groups exhibit the same spike spectra of topological origin. This result is illustrated in fig.3 where it is shown a PSH, and an MPSH computed with 50 simulated catalogs in this 3-torus. All catalogs used here are comparable to those used in the simulations performed in Sec.3 for an observed universe of radius $R_{1}$, and have approximately 240 sources. We can note that there is no relevant difference between plots corresponding to $M$ and its minimal covering 3-torus even after reducing the noise by calculating the mean over 50 catalogs. Thus, looking at any of these plots one cannot say whether it comes from a catalog of sources in a $\mathcal{G}_{6}$ universe or in its 3-torus minimal covering: topological spikes are not enough to distinguish between these two flat manifolds because they have the same set of Clifford translations in their covering groups.

To evince the topological signature of non-translational isometries of $M$ in $\mathrm{CC}$, and so be able to distinguish between $M$ and any other manifold, we make use of (2.14) and (2.15). Fig.4 shows the graph of $\Phi_{\text {exp }}^{\text {torus }}\left(s_{i}\right)$ given by eq.(2.15) together with (2.16) for the case of an observed universe of radius $R_{1}=\sqrt{2}$. The comparison between the EPSH of fig. 4 and the MPSH shown in fig.3b makes apparent the strength of the MPSH procedure. In both fig. 4 and fig. 5 we have reduced the number of sources from 240 to 120 to enhance the amplitude of the topological signature. In these figures we have also incremented the width of the bins from 0.01 to 0.02 just to keep the amplitude of the spikes as in the previous simulations. Figs.5a and $5 \mathrm{~b}$ are plots of eq.(2.14). The first plot corresponds to eq.(2.14) with an MPSH, $\left\langle\Phi\left(s_{i}\right)>\right.$, for the 3-torus while the second corresponds to eq. 2.14) with an MPSH for $M$. Both MPSH's were built with 
5000 catalogs. Since the covering groups of this 3 -torus and that of $M$ have the same translations, PSH's for these two manifolds would exhibit identical spike spectra; so the differences given by eq.(2.14) yield the topological signature of eventual non-translational isometries plus some statistical noise. Fig.5a is essentially noise, as expected, since there are no non-translational isometries in the covering group of the 3-torus; while fig.5b evinces the topological signature of non-translational isometries of $\Gamma$ (the covering group of $M$ ). In fig.5b, the amplitude of the topological signature of non-translational isometries is roughly one order of magnitude smaller than the amplitude and the statistical noise of the PSH's in fig.1, so this signature could hardly be eventually extracted from a single PSH constructed with a real catalog. We have been able to evince this tiny topological signature in our simulations because we have succeeded in reducing the statistical noise by two orders of magnitude using the MPSH technique. Incidentally, if there is any other component to the topological signature in PSH's of 3-tori (i.e. other than spikes), its amplitude must be at least three orders of magnitude smaller than that of a PSH, as shown in fig.5a.

One can obtain a better understanding of the structural features exhibited in fig.5b, in particular the exact locations of the "jumps", by noting that, since $\Gamma$ acts freely and discontinuously on euclidean space, there is a minimum non-null pair separation $|g|_{\text {min }}$ among all $g$-pairs, for each $g \in \widetilde{\Gamma}$. Thus, the terms $\Phi_{e x p}^{g}\left(s_{i}\right)$, which appear in the definition (2.11) of the topological signature of non-translational isometries, are zero until the $i$-th bin given by the condition $|g|_{\text {min }}^{2} \in J_{i}$. As reported in [9] (see fig.2a and fig.2b therein), these terms start with a jump at this $i$-th bin, therefore one expects that the topological signature of non-translational isometries presents such jumps at well defined positions that we will calculate in what follows.

Note that all non-translational isometries of $\Gamma$ can be grouped in the following three categories according to whether the parameters $l$ and $m$ are even (E) or odd (O) (see Appendix A)

Type OE $l=2 k_{1}+1$ and $m=2 k_{2}$,

Type EO $l=2 k_{1}$ and $m=2 k_{2}+1$, and

Type OO $l=2 k_{1}+1$ and $m=2 k_{2}+1$,

where $k_{1}$ and $k_{2}$ are integers.

Straightforward calculations yield

$$
\begin{aligned}
\left|\rho\left(2 k_{1}+1,2 k_{2}, n\right)\right|_{\text {min }}^{2} & =\left(2 k_{1}+1\right)^{2}, \\
\left|\mu\left(2 k_{1}+1,2 k_{2}, n\right)\right|_{\text {min }}^{2} & =\left(2 k_{1}+1\right)^{2},
\end{aligned}
$$




\begin{tabular}{|c|c|c|c|c|}
\hline$|g|_{\text {min }}^{2}$ & Type & $k_{1}$ & $k_{2}+n$ & $n$ \\
\hline \hline \multirow{4}{*}{0.5} & $\mathrm{EO}$ & - & $0,-1$ & - \\
\cline { 2 - 5 } & $\mathrm{OO}(\rho)$ & - & - & 0,1 \\
\cline { 2 - 5 } & $\mathrm{OO}(\mu)$ & - & - & $0,-1$ \\
\hline 1 & $\mathrm{OE}$ & $0,-1$ & - & - \\
\hline \multirow{4}{*}{4.5} & $\mathrm{EO}$ & - & $1,-2$ & - \\
\cline { 2 - 5 } & $\mathrm{OO}(\rho)$ & - & - & $-1,2$ \\
\cline { 2 - 5 } & $\mathrm{OO}(\mu)$ & - & - & $1,-2$ \\
\hline 9 & $\mathrm{OE}$ & $1,-2$ & - & - \\
\hline \multirow{4}{*}{12.5} & $\mathrm{EO}$ & - & $2,-3$ & - \\
\cline { 2 - 5 } & $\mathrm{OO}(\rho)$ & - & - & $-2,3$ \\
\cline { 2 - 5 } & $\mathrm{OO}(\mu)$ & - & - & $2,-3$ \\
\hline
\end{tabular}

Table 1: The first five values for the squared minimum distance $|g|_{\min }^{2}$ of $g$-pairs, and specifications of the type of isometries together with the values of the corresponding parameters which give rise to them.

for type OE isometries;

$$
\begin{aligned}
& \left|\rho\left(2 k_{1}, 2 k_{2}+1, n\right)\right|_{\text {min }}^{2}=2\left(k_{2}+n+1 / 2\right)^{2}, \\
& \left|\mu\left(2 k_{1}, 2 k_{2}+1, n\right)\right|_{\text {min }}^{2}=2\left(k_{2}+n+1 / 2\right)^{2},
\end{aligned}
$$

for type EO isometries; and

$$
\begin{aligned}
\left|\rho\left(2 k_{1}+1,2 k_{2}+1, n\right)\right|_{\min }^{2} & =2(n-1 / 2)^{2}, \\
\left|\mu\left(2 k_{1}+1,2 k_{2}+1, n\right)\right|_{\min }^{2} & =2(n+1 / 2)^{2} .
\end{aligned}
$$

for type $\mathrm{OO}$ isometries. Here $\rho(l, m, n)$ and $\mu(l, m, n)$ are given by equation (A.1).

Now it can easily be seen that the non-translational contributions to the topological signature sharply start at $s_{i}=0.5,1,4.5,9,12.5 \ldots$ (see table — for clarification). An observable universe for a full sky covering survey and radius $R_{1}$ allows non-null contributions from non-translational isometries with $|g|_{\text {min }}^{2}<4 R_{1}^{2}=8$, and so its topological signature must present "jumps" at $s_{i}=0.5,1$ and 4.5 only, in agreement with fig.5b.

To close this section we emphasize that the MPSH technique is a suitable approach to obtain the topological signatures only when one is dealing with simulated catalogs. Note however that the ultimate step in most of such statistical approaches to extract the topological signature is the comparison of the graphs (signature) obtained from simulated 
catalogs against similar graphs generated from real catalogs. To do so one clearly has to have the simulated patterns of the topological signatures of the manifolds, which can be achieved by the MPSH approach discussed in this work.

\section{$5 \quad$ Final remarks}

In this letter we have studied, through simulations concerning closed flat models of our Universe, some general results obtained in Ref. [2], namely, that topological spikes in PSH's arise from translations, while non-translational isometries manifest as slight deformations of the EPSH of the corresponding simply connected case.

We have used the technique of taking means of PSH's, described in Ref. [2], to reduce the noise to a level that allows (i) to distinguish between topological and statistical spikes, and (ii) to evince the shape of the topological signature of non-translational isometries. In particular, we have shown explicitly that topological spikes for a cubic manifold $M$ of class $\mathcal{G}_{6}$ with $L=1$, appear only at even integers in plots of $n(d)$ vs. $d^{2}$, so the spike at $(d / L)^{2}=5$ reported in Fig.1 of Ref.[7] is indeed due to statistical fluctuations, in agreement with the theoretical conclusion we have derived from the results of [2].

Identifying the shape of the topological signature of non-translational isometries is possible only through a drastic reduction of the statistical noise followed by the elimination of the uncorrelated part of the EPSH together with the topological spikes. This last step has been attained by subtracting the EPSH of the minimal 3-torus that covers $M$ from an MPSH built from $M$ and with small enough noise. In doing so we have shown that the topological signature of non-translational isometries is formed by broad and tiny distributions, each one beginning with a "jump" at $|g|_{\text {min }}^{2}$, for some isometry $g$.

The methods employed in this work allowed the study of the nature of the topological signature in CC of euclidean non-translational isometries, but can be equally applied to any of the other two geometries of constant curvature. Note, however, that these methods may not be useful in applications of CC to real catalogs, since it is impossible in practice to construct thousands, or even hundreds, of comparable catalogs of real cosmic sources. For an implementation of CC along these lines for use with real catalogs, a novel technique for reducing statistical noise with just one catalog has to be developed. We emphasize however that, despite the use of MPSH's be restricted to simulated catalogs, it is a suitable approach for studying the topological signature of non-translational isometries in PSH's, and thus it is a very important tool for the understanding of the method of CC. Clearly, without the understanding which arises from the MPSH approach to CC it would be quite 
difficult to establish the applicability of the crystallographic method as well as to devise alternative methods in a systematic way.

\section{Acknowledgements}

We thank the Brazilian scientific agencies CNPq and CAPES for financial support.

\section{Appendix A}

In this Appendix we show explicitly that there are no topological spikes at odd integer positions in PSH's constructed from models using $M$ (given by the covering group $\Gamma$ generated by (3.1)) as spatial sections of spacetime. From the relations $\beta^{2}=\delta^{2}, \beta \alpha=$ $\alpha^{-1} \beta, \delta \alpha=\alpha^{-1} \delta$, and $\beta \alpha^{-1}=\alpha \delta^{-1}$, and from the fact that $\alpha^{2}, \beta^{2}, \beta \delta$ and $\delta \beta$ are translations, and so they commute, one can write any covering isometry of $M$ in one of the two canonical forms

$$
\begin{aligned}
& \rho(l, m, n)=\alpha^{l} \beta^{m}(\beta \delta)^{n}, \\
& \mu(l, m, n)=\alpha^{l} \beta^{m}(\delta \beta)^{n},
\end{aligned}
$$

where $l, m$ and $n$ are integers, and $n \geq 0$. For all $l$ and $m$ we have that $\rho(l, m, 0)=$ $\mu(l, m, 0)$, while for $n \neq 0, \rho(l, m, n) \neq \mu(l, m, n)$.

The translations in $\Gamma$ are those isometries with $l$ and $m$ even, regardless of the value of $n$. Explicitly we have

$$
\begin{aligned}
& \rho\left(2 k_{1}, 2 k_{2}, n\right)(x, y, z)=\left(x+2 k_{1}, y+k_{2}+2 n, z+k_{2}\right), \\
& \mu\left(2 k_{1}, 2 k_{2}, n\right)(x, y, z)=\left(x+2 k_{1}, y+k_{2}, z+k_{2}+2 n\right),
\end{aligned}
$$

where $k_{1}$ and $k_{2}$ are integers, and so

$$
\left|\rho\left(2 k_{1}, 2 k_{2}, n\right)\right|^{2}=\left|\mu\left(2 k_{1}, 2 k_{2}, n\right)\right|^{2}=4 k_{1}^{2}+k_{2}^{2}+\left(k_{2}+2 n\right)^{2} .
$$

Equation (A.2) gives the position of any potential topological spike in PSH's based on models of the universe with $M$ as a spatial section of spacetime. Whether a given spike will appear depends on the shape and size of the observed universe, i.e. on the shape and deepness of the region scanned by the astronomical survey used to construct the catalog. However, by writing Eq.(A.2) in the form

$$
\left|\rho\left(2 k_{1}, 2 k_{2}, n\right)\right|^{2}=2\left[2 k_{1}^{2}+\left(k_{2}+n\right)^{2}+n^{2}\right],
$$


and an identical expression for $\left|\mu\left(2 k_{1}, 2 k_{2}, n\right)\right|^{2}$, one can conclude that there are no spikes of topological origin at odd integer positions, since $\left|\rho\left(2 k_{1}, 2 k_{2}, n\right)\right|^{2}$ and $\left|\mu\left(2 k_{1}, 2 k_{2}, n\right)\right|^{2}$ take always even integer values. In particular, there is no topological spike at $s=5$. This is in agreement with the simulations which gave rise to figs. 1 and 2, and shows that the spike found by Fagundes and Gausmann at $(d / L)^{2}=5$ is of statistical origin.

\section{Captions for the figures}

Figure 1. PSH's $\Phi\left(s_{i}\right)$ for two $\mathcal{G}_{6}$ universes. In (a) the corresponding observed universe is a ball of radius $R_{1}=\sqrt{2}$, in (b) $R_{2}=5 / 6$. In both cases the catalogs have approximately 240 sources. The intervals $\left(0,4 R^{2}\right]$ have been subdivided in bins of width 0.01. In (a) the PSH presents no apparent spike at $s=5$.

Figure 2. MPSH's $<\Phi\left(s_{i}\right)>$ built with 50 simulated comparable catalogs with approximately 240 sources for the two $\mathcal{G}_{6}$ universes of fig. 1 . The statistical noise has been considerably reduced so that it becomes apparent that there is no topological spike at $s=5$ in (a), whereas in (b) there is a small spike at $s=2$ that is masked by statistical fluctuations in the PSH of fig.1b. The intervals $\left(0,4 R^{2}\right]$ have been subdivided in bins of width 0.01 .

Figure 3. Part (a) is a PSH $\Phi\left(s_{i}\right)$ and part (b) is a MPSH $<\Phi\left(s_{i}\right)>$ for the 3-torus universe which covers the $\mathcal{G}_{6}$ universe of figs.1a and 2a. All catalogs used here are comparable to those used for figs.1a and 2a, and have approximately 240 sources. The intervals $\left(0,4 R^{2}\right]$ have been subdivided in bins of width 0.01 . There is no relevant difference between graphs corresponding to the $\mathcal{G}_{6}$ model and its minimal covering 3-torus, illustrating that topological spikes are not enough to distinguish between these two flat manifolds.

Figure 4. An EPSH $\Phi_{\text {exp }}\left(s_{i}\right)$ given by (2.15) for the 3-torus whose PSH $\Phi\left(s_{i}\right)$ is shown in fig.3. The comparison between the EPSH of the present figure with the MPSH of fig. $3 \mathrm{~b}$ makes apparent the suitability and strength of the MPSH procedure.

Figure 5. MPSH's corresponding to the topological signature of non-translational isometries given by (2.14). Part (a) corresponds to the 3-torus of fig.3 and fig.4, while (b) corresponds to the $\mathcal{G}_{6}$ manifold of figs.1 and 2. Both MPSH's were built with 5000 catalogs of approximately 120 sources, and with bins of width 0.02 . While (a) 
exhibits essentially statistical noise as expected, (b) shows the topological signature of non-translational isometries of $\mathcal{G}_{6}$.

\section{References}

[1] R. Lehoucq, M. Lachièze-Rey \& J.-P. Luminet, Astron. Astrophys. 313, 339 (1996).

[2] G.I. Gomero, A.F.F. Teixeira, M.J. Rebouças \& A. Bernui, Spikes in Cosmic Crystallography, gr-qc/9811038 (1998).

[3] G.I. Gomero, M.J. Rebouças \& A.F.F. Teixeira, A Signature for the Shape of the Universe, Int. J. Mod. Phys. D, in press (2000).

[4] R. Lehoucq, J.-P. Luminet \& J.-P. Uzan, Astron. Astrophys. 344, 735 (1999).

[5] H.V. Fagundes \& E. Gausmann, Cosmic Crystallography in Compact Hyperbolic Universes, astro-ph/9811368 (1998).

[6] J.-P. Uzan, R. Lehoucq, \& J.-P. Luminet, Astron. Astrophys. 351, 766 (1999).

[7] H.V. Fagundes \& E. Gausmann, Phys. Lett. A 238, 235 (1998).

[8] J.A. Wolf, Spaces of Constant Curvature, fifth ed., Publish or Perish Inc., Delaware (1984).

[9] G.I. Gomero, M.J. Rebouças \& A.F.F. Teixeira, A topological signature in cosmic topology, gr-qc/9911049 (1999).

[10] A. Bernui \& A.F.F. Teixeira, Cosmic crystallography: three multi-purpose functions, astro-ph/9904180 (1999).

[11] M.J. Rebouças Distinguishing Marks of Simply-Connected Universes, gr-qc/000740. Int. J. Mod. Phys. D 9, in press (2000).

[12] G.I. Gomero, Fundamental Polyhedron and Glueing Data for the Sixth Euclidean Compact Orientable 3-manifold, preprint CBPF-NF-049/97 (1997).

[13] A. Bernui, G.I. Gomero, M.J. Rebouças \& A.F.F. Teixeira, Phys. Rev. D 57, 4699 (1998). 

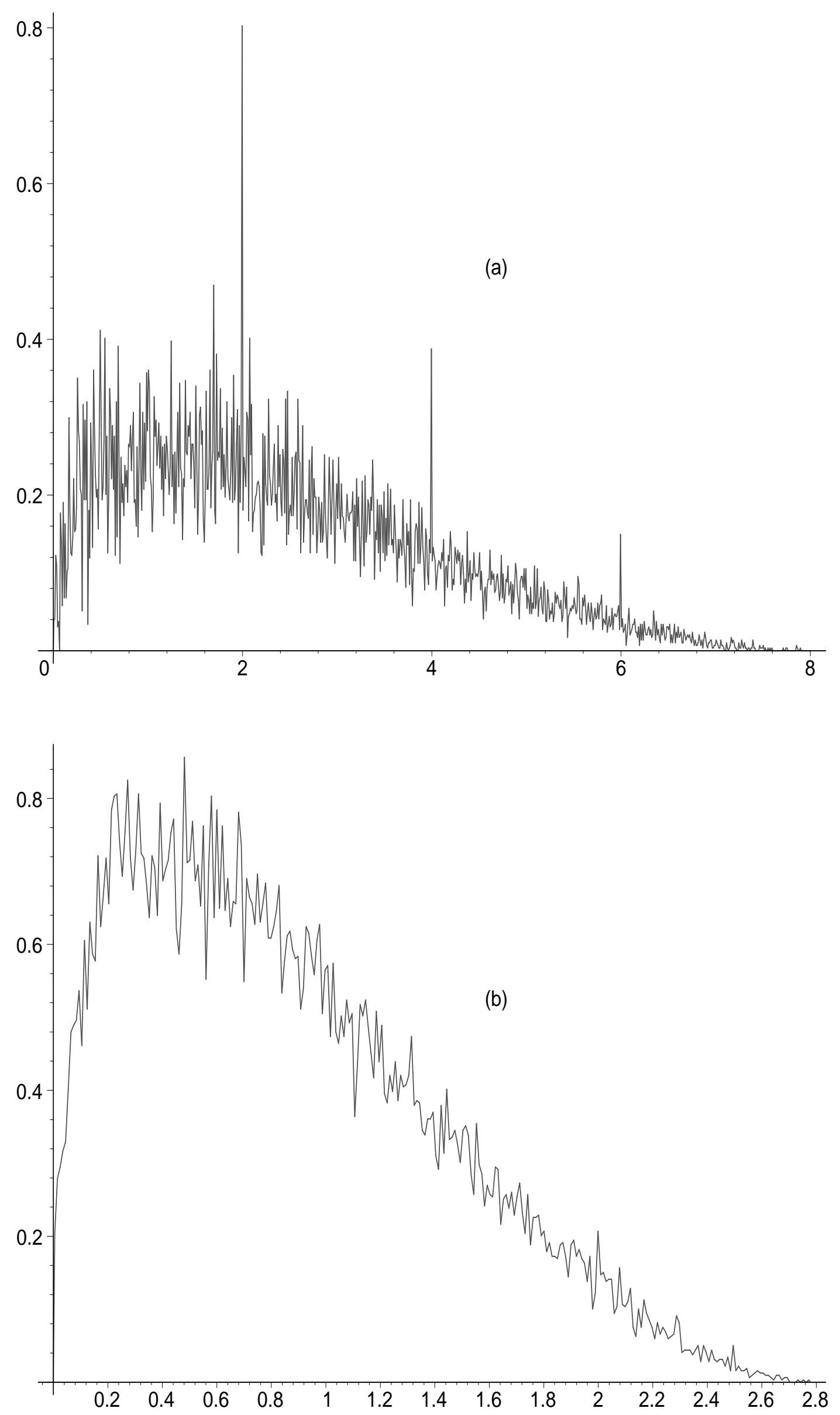

Figure 1 

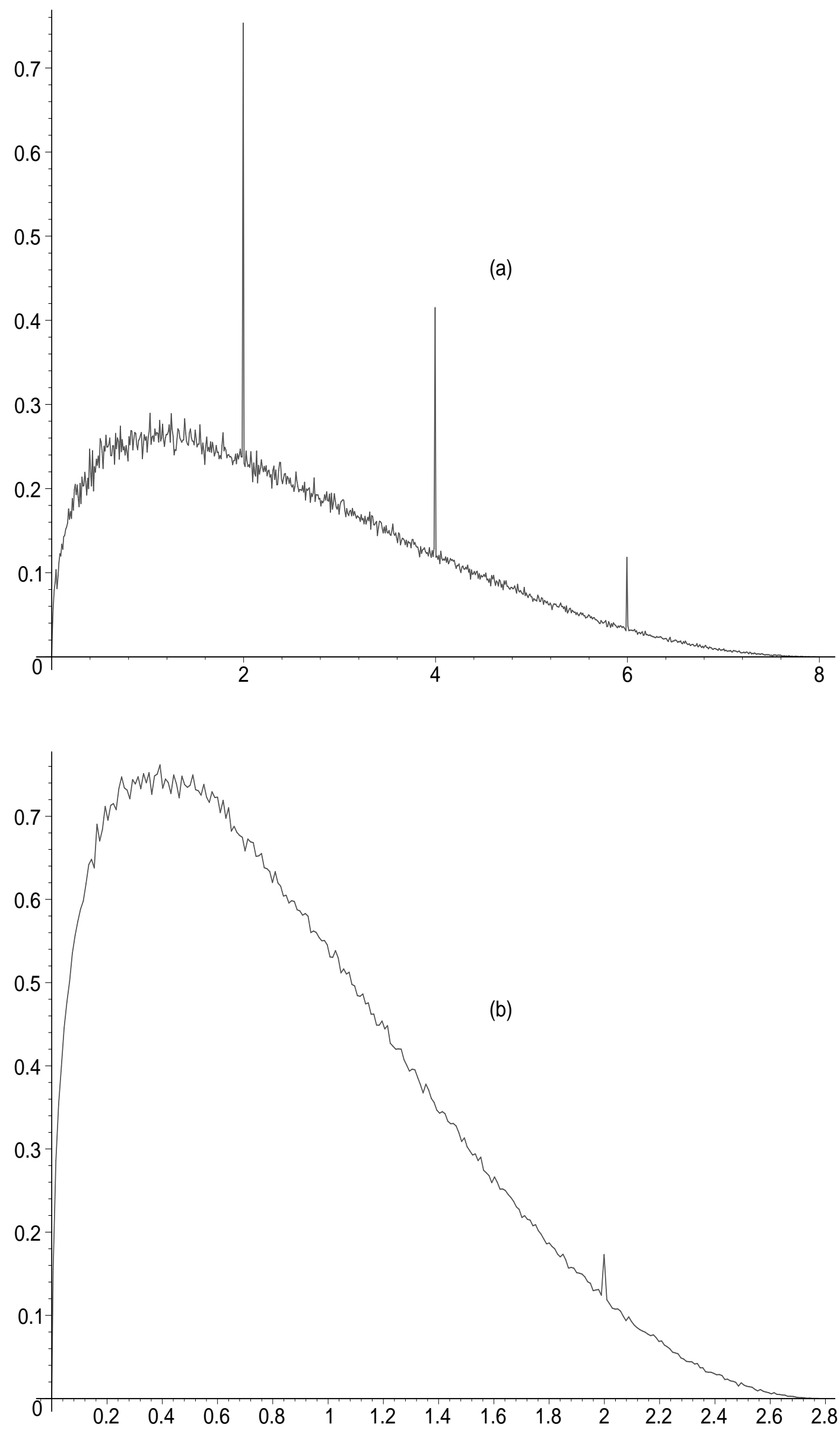

Figure 2 

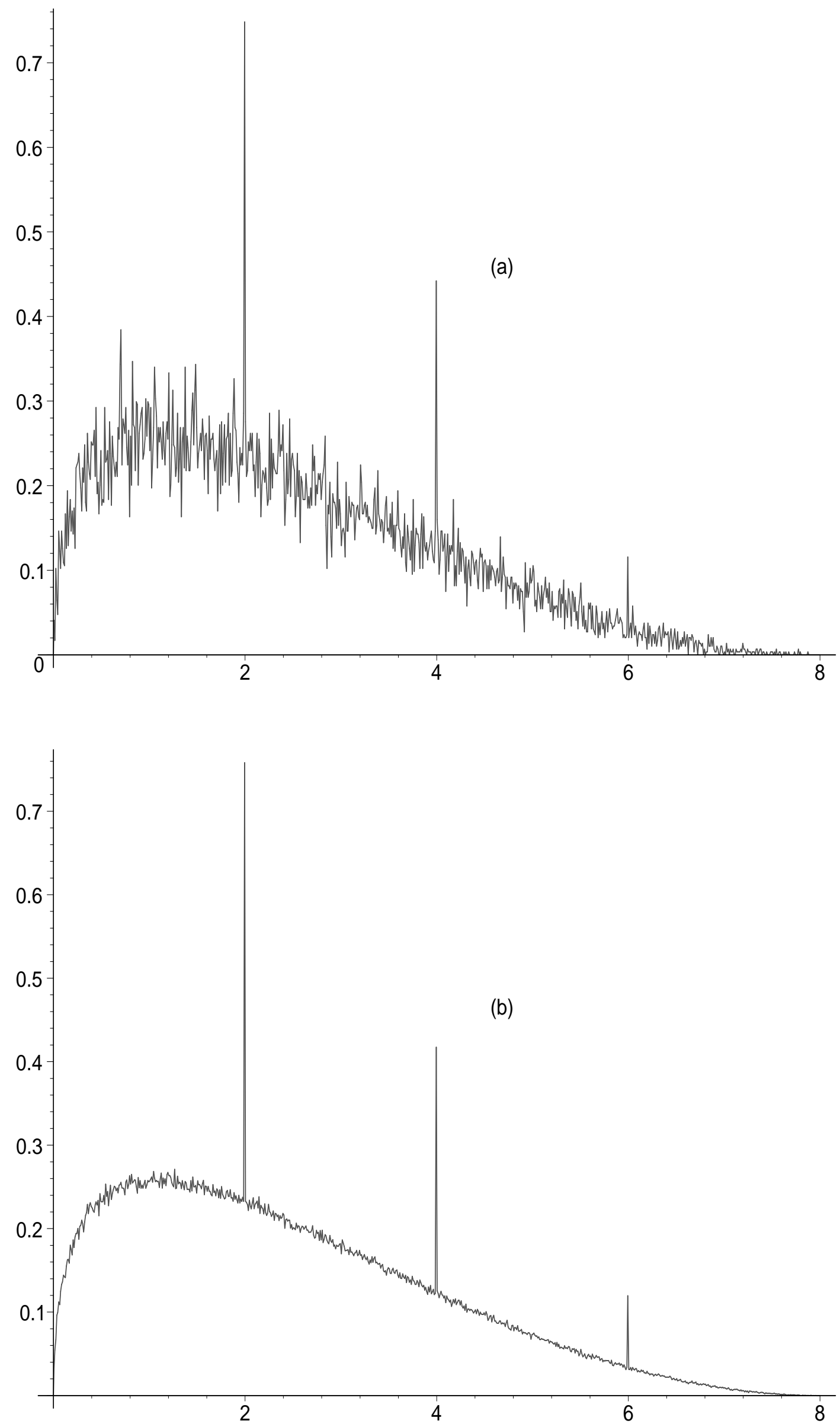

Figure 3 


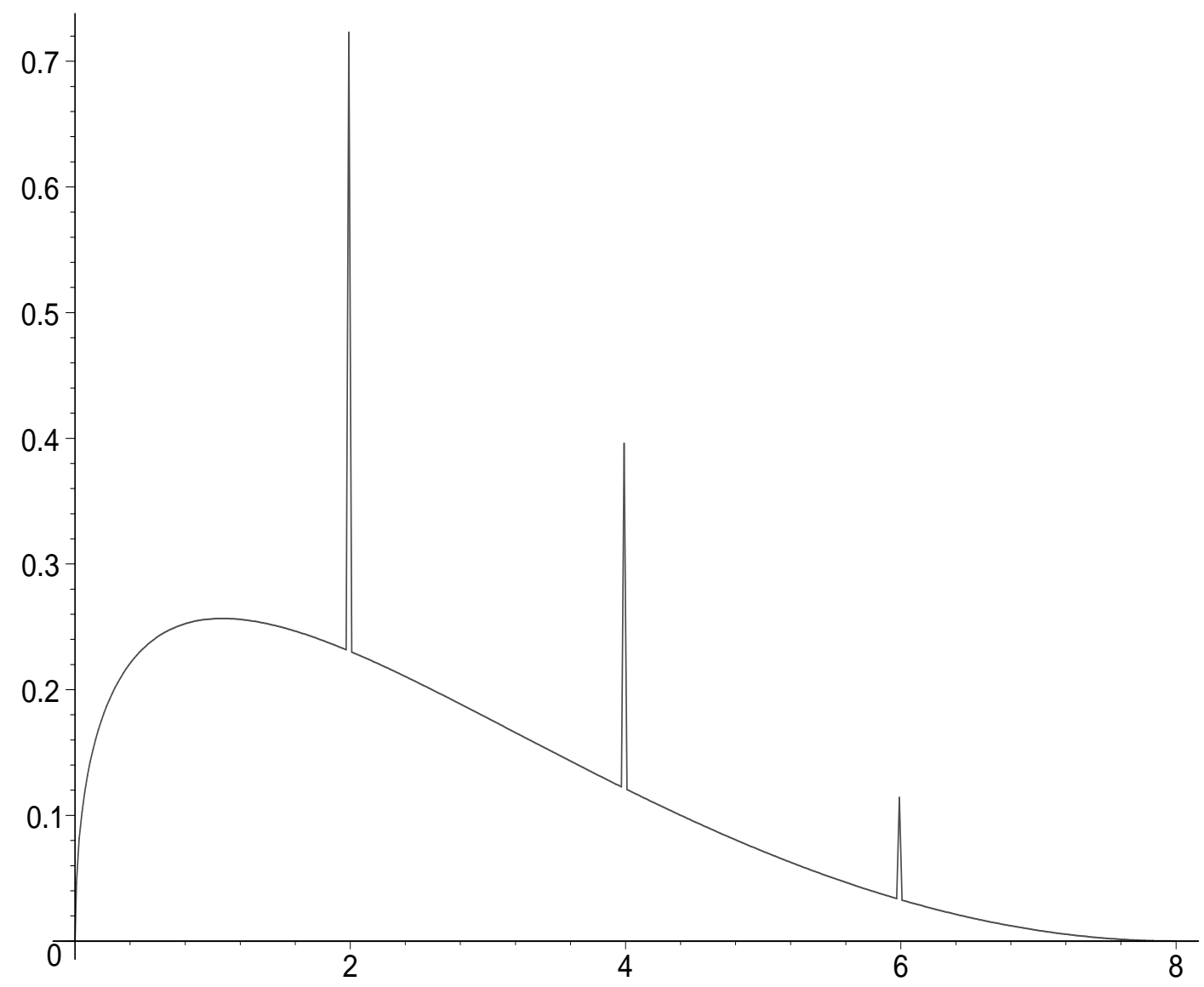

Figure 4 

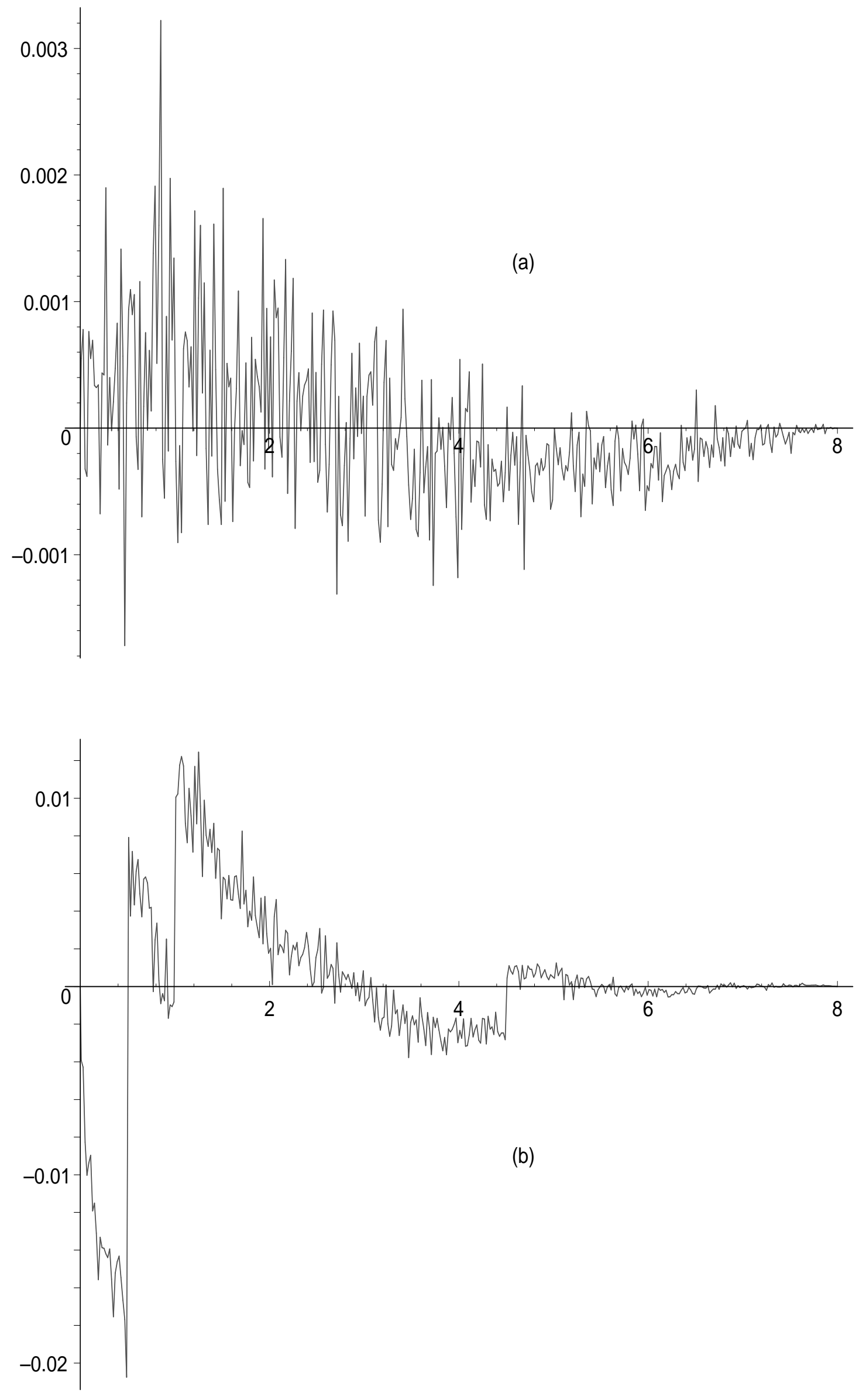

Figure 5 